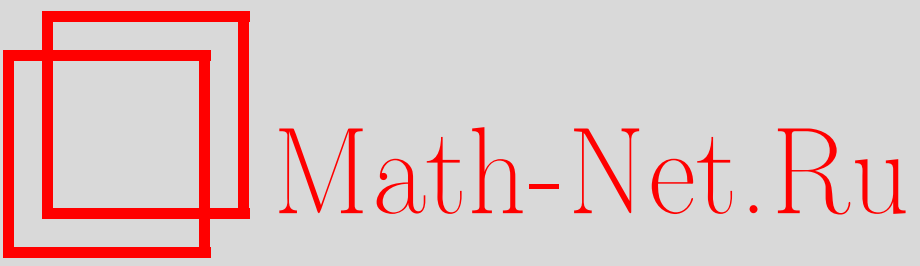

С. А. Кудрявцев, Об исключительных терминальных особенностях, УМН, 2001, том 56, выпуск 1, 165-166

DOI: https://doi.org/10.4213/rm366

Использование Общероссийского математического портала Math-Net.Ru подразумевает, что вы прочитали и согласны с пользовательским соглашением

http://www.mathnet.ru/rus/agreement

Параметры загрузки:

IP : 3.89 .197 .203

26 апреля 2023 г., 16:34:06 


\title{
ОБ ИСКЛЮЧИТЕЛЬНЫХ ТЕРМИНАЛЬНЫХ ОСОБЕННОСТЯХ
}

\author{
С. А. КудРявцеВ
}

Важность изучения исключительных особенностей следует из следующего наблюдения: если особенность не исключительна, то для нее можно найти "хороший" дивизор из линейной системы $\left|-n K_{X}\right|$ для небольших значений $n$. Например, для двухмерных логканонических особенностей $n \in\{1,2\}[1 ; 5.2]$, а в трехмерном случае $n \in\{1,2,3,4,6\}$ [2; 7.1]. А исключителшные особенности "ограниченны" и можно дать их подробное описание. В данной работе строятся первые примеры исключительных терминальных особенностей.

Автор очень признателен В. А. Исковских, Ю. Г. Прохорову за проявленный интерес к работе.

1. Предварительные сведения. Все многообразия рассматриваются над полем комплексных чисел $\mathbb{C}$. Основные определения, обозначения и понятия приведены в [3], [4].

ОПРЕДЕЛЕнИЕ 1.1. Пусть $(X / Z \ni P, D)$ - стягивание многообразий. $\mathbb{Q}$-дополнением для этого стягивания называется эффективньй $\mathbb{Q}$-дивизор $D^{\prime}$ такой, что $D^{\prime} \geqslant D$, дивизор $K_{X}+D^{\prime}$ - логканоничен и $K_{X}+D^{\prime} \sim_{\mathbb{Q}} 0$.

ОПРЕДЕЛЕНИЕ 1.2 . Пусть $(X / Z \ni P, D)$ - стягивание многообразий. Если $\operatorname{dim} Z \neq 0$, то стягивание называется исключительнылм, если для любого $\mathbb{Q}$-дополнения $D^{\prime}$ существует не более одного дивизора $E$ (не обязательно исключительного) такого, что $a\left(E, D^{\prime}\right)=-1$. Если $\operatorname{dim} Z=0$, то стягивание назьвается исключительныл, если для любого $\mathbb{Q}$-дополнения $D^{\prime}$ пара $\left(X, D^{\prime}\right)$ логтерминальна по Кавамате.

ПримеР 1.3. Если для особенности $(X \ni P)$ существует эффективньй дивизор $H$ такой, что пара $(X, H)$ - логканонична и $\llcorner H\lrcorner \neq 0$, то особенность не исключительна. Поэтому трехмерная терминальная особенность не исключительна, так как в антиканонической линейной системе $\left|-K_{X}\right|$ существует дивизор с дювалевскими особенностями $[5 ; 6.4]$.

ОПредЕЛЕнИЕ 1.4. Пусть $(X \ni P)$ - логтерминальная особенность, и пусть $f: Y \rightarrow X$ некоторое ее раздутие. Предположим, что исключительное множество $f$ состоит из одного неприводимого дивизора $E$. Тогда $f:(Y, E) \rightarrow(X \ni P)$ называется чисто логтерминальныц раздутием, если дивизор $K_{Y}+E$ чисто логтерминален и $-\left(K_{Y}+E\right) f$-обилен.

ЗАмЕчаниЕ 1.5. Чисто логтерминалњное раздутие всегда существует $[6 ; 1.5]$.

Теорема $1.6[7 ; 4.9]$. Пусть $(X \ni P)$ - логтерминальная особенность, и пусть $f:(Y, E)$ $\rightarrow X$ - чисто логтерминальное раздутие $X$. Тогда следующие утверждения әквивалентны:

(1) особенность $(X \ni P)$ - исключительная;

(2) логмногообразие $\left(E, \operatorname{Diff}_{E}(0)\right)$ - исключительное.

\section{2. Примеры исключительных терминальных особенностей.}

Tеорема 2.1. Пусть $(f=0,0)=\left(x_{1}^{a_{1}}+x_{2}^{a_{2}}+x_{3}^{a_{3}}+x_{4}^{a_{4}}+x_{5}^{a_{5}}=0,0\right) \subset\left(\mathbb{C}^{5}, 0\right)$ - четырехмерные гиперповерхностные особенности, где $\left(a_{1}, \ldots, a_{5}\right)=(2,3,11,17,19)$, $(2,3,11,17,23),(2,3,11,17,25),(2,3,11,17,29),(2,5,7,9,11),(2,5,7,9,13)$. Тогда они терминальны и исключительны.

ДокАЗАтельство. Рассмотрим первую особенность. Сначала докажем, что эта особенность терминальна. Так как она невырожденная, то для нее существует вложенное торическое логразрешение [8]. Поэтому удобно доказать, что $a_{\mathbf{p}}=\langle\mathbf{p}, 1\rangle-\mathbf{p}(f)-1 \geqslant 1$ для всех $\mathbf{p}$, где $\mathbf{p}-$ ненулевой вектор с целочисленными неотрицательными координатами и $\mathbf{p}(f)=\min _{x} \in f\langle\mathbf{p}, m\rangle$. Проще всего это сделать следующим образом. Пусть $h(d)=\left\ulcorner\frac{d}{2}\right\urcorner+\left\ulcorner\frac{d}{3}\right\urcorner+\left\ulcorner\frac{d}{11}\right\urcorner+\left\ulcorner\frac{d}{17}\right\urcorner+\left\ulcorner\frac{d}{19}\right\urcorner-d-1$.

Работа выполнена при частичной поддержке грантов РФФИ № 99-01-01132, ведущих научных школ № 96-15-96146 и грантов INTAS-OPEN № 93-2805 и № 97-2072. 
Положим $d=\mathbf{p}(f)$, тогда $h(d) \leqslant a_{\mathbf{p}}$. Отсюда достаточно доказать, что $h(d) \geqslant 1$ для всех $1 \leqslant d \leqslant 2 \cdot 3 \cdot 11 \cdot 17 \cdot 19$. Последнее элементарно проверить с помощью компьютерной программы.

Взвешенное раздутие $\mathbb{C}^{5}$ с весами, пропорциональными $\left(\frac{1}{2}, \frac{1}{3}, \frac{1}{11}, \frac{1}{17}, \frac{1}{19}\right)$, индуцирует чисто логтерминальное раздутие нашей особенности. Полученное многообразие лог Фано $\left(E, \operatorname{Diff}_{E}(0)\right)$ - это

$$
(\underbrace{\sum_{i=1}^{5} x_{i} \subset \mathbb{P}(1,1,1,1,1)}_{\mathbb{P}^{3}}, \frac{1}{2} H_{1}+\frac{2}{3} H_{2}+\frac{10}{11} H_{3}+\frac{16}{17} H_{4}+\frac{18}{19} H_{5}),
$$

где $H_{i}=\left\{x_{i}=0\right\}$.

Пусть $D=\sum d_{i} D_{i}+\sum h_{i} H_{i}-$ произвольное $\mathbb{Q}$-дополнение для этой пары. В данном случае легко проверить, что $d_{i}<1$ и $h_{i}<1$ для всех $i$. Если любой $D_{i}$ является гиперплоским сечением, то легко проверить, что дивизор $K_{\mathbb{P} 3}+D$ - логтерминален по Кавамате. Докажем, что всегда пара $\left(\mathbb{P}^{3}, D\right)$ логтерминальна по Кавамате. Этот вопрос локальньй, поэтому рассмотрим произволшную карту $\mathbb{C}_{y_{1}}^{3}, y_{2}, y_{3}$. Пусть $D=\Delta+D^{\prime}$, где $\Delta$ состоит из гиперплоских сечений и $D^{\prime}=\sum d_{i}\left\{f_{i}=0\right\}$. Пусть минимальная степень $f_{i}$ равняется $n_{i}$. Рассматривая замену координат в $\mathbb{C}^{3}$, без ограничения общности можно считать, что $f_{i}=y_{1}^{n_{i}}+\cdots$. Рассмотрим деформацию $F_{t}^{\prime}=\sum d_{i} t^{-n_{i}} f_{i}\left(t y_{1}, t^{2} y_{2}, t^{2} y_{3}\right)$. При $t=0$ имеем $D_{0}^{\prime}=\left\{F_{0}^{\prime}=0\right\}=\sum d_{i}\left\{y_{1}^{n_{i}}=0\right\}=$ $\sum d_{i} n_{i}\left\{y_{1}=0\right\}$, а при малых $t \neq 0$ следует, что $D_{t}^{\prime}=\left\{F_{t}^{\prime}=0\right\}=D^{\prime}$. Из [9; 8.6] имеем, что $c\left(\mathbb{C}^{3}, \Delta+D_{0}^{\prime}\right) \leqslant c\left(\mathbb{C}^{3}, \Delta+D_{t}^{\prime}\right)=c\left(\mathbb{C}^{3}, \Delta+D^{\prime}\right)$, где $c$ - логканонический порог. Так как каждая компонента $\operatorname{Supp}\left(\Delta+D_{0}^{\prime}\right)$ является гиперплоским сечением, то пара $\left(\mathbb{C}^{3}, \Delta+D_{0}^{\prime}\right)$ логтерминална по Кавамате. Поэтому пара $\left(\mathbb{C}^{3}, \Delta+D^{\prime}\right)$ тем более логтерминална по Кавамате. По критерию 1.6 особенность исключительная. Дивизор $\frac{1}{22}\left(\left\llcorner 23 \cdot \operatorname{Diff}_{E}(0)\right\lrcorner\right)$ дает 22 -дополнение минимального индекса. Осталњные особенности рассматриваются аналогичным образом. Они соответственно 24-, 34-, 34-, 22-, 28-дополняемы.

ЗАмЕчАНИЕ 2.2. Из гипотезы о том, что минималњный индекс дополняемости поверхностей лог Дель-Пецо со стандартными коэффициентами не превосходит 66 [2], следует ограничение на вид $\mathbb{Q}$-дополнения $D=\sum d_{i} D_{i}$ для 4 -мерных особенностей. Пусть $d_{i}=r_{i} / q_{i}$, тогда $q_{i} \leqslant 66$. Это позволяет рассматривать конечное число вариантов для $D$.

ГИПОТЕЗА 2.3. Пусть ( $X \ni P)$ - п-мерная каноническая (логканоническая) гиперповерхностная особенность. Пусть $f$-логразрешение $u \min \{a(E, 0) \mid f(E)=P\} \geqslant n-2$. Тогда существует гиперплоское сечение $H$ такое, что пара $(X, H)$ чисто логтерминальна (логканонична). Отсюда следует, что особенность не слабо исключительна [7; 4.8] (не исключительна).

\section{СПИСОК ЛИТЕРАТУРЫ}

[1] В. В. Шокуров // Изв. АН СССР. Сер. матем. 1992. Т. 56. С. 105-203. [2] V. V. Shokurov. Complements on surfaces // e-print math.AG/9711024. [3] J. Kollar (ed.). Flips and Abundance for Algebraic Threefolds. Paris: Societé Mathématique de France, 1992. (Astérisque. V. 211.) [4] Yu. G. Prokhorov. Lectures on complements on log surfaces // e-print math.AG/ 9912111. [5] M. Reid // Proc. Sympos. Pure Math. 1987. V. 46. P. 343-416. [6] C. А. Кудрявцев. О чисто логтерминальных раздутиях // Матем. заметки. (в печати). [7] Yu. G. Prokhorov // Algebra: Proceedeings of the International Conference on the Occasion of the 90th birthday of A. G. Kurosh, Moscow, Russia, May 25-30, 1998 / ed. Yu. Bahturin. Berlin: de Gruyter, 2000. P. 301-317. [8] A. N. Varchenko // Invent. Math. 1976. V. 37. P. 253-262. [9] J. Kollár // Proc. Sympos. Pure Math. 1995. V. 62. P. 221-287. 